\title{
CTA Collateral Status and Response to Recanalization in Patients with Acute Ischemic Stroke
}

\author{
V. Nambiar, S.I. Sohn, M.A. Almekhlafi, H.W. Chang, S. Mishra, E. Qazi, M. Eesa, A.M. Demchuk, M. Goyal, M.D. Hill, and B.K. Menon
}

\begin{abstract}
BACKGROUND AND PURPOSE: Collateral status at baseline is an independent determinant of clinical outcome among patients with acute ischemic stroke. We sought to identify whether the association between recanalization after intra-arterial acute stroke therapy and favorable clinical response is modified by the presence of good collateral flow assessed on baseline CTA.
\end{abstract}

MATERIALS AND METHODS: Data are from the Keimyung Stroke Registry, a prospective cohort study of patients with acute ischemic stroke from Daegu, South Korea. Patients with M1 segment MCA with or without intracranial ICA occlusions on baseline CTA from May 2004 to July 2009 who also had baseline MR imaging were included. Two readers blinded to all clinical information assessed baseline and follow-up imaging. Leptomeningeal collaterals on baseline CTA were assessed by consensus by use of the regional leptomeningeal score.

RESULTS: Among 84 patients (mean age, $65.2 \pm 13.2$ years; median NIHSS score, 14; interquartile range, 8.5 ), median time from stroke onset to initial MR imaging was 164 minutes. $\mathrm{TICl} 2 \mathrm{~b}-3$ recanalization was achieved in $38.1 \%$ of patients and mRS $0-2$ at 90 days in $35.8 \%$ of patients. In a multivariable model, the interaction between collateral status and recanalization was significant. Only patients with intermediate or good collaterals who recanalized showed a statistically significant association with good clinical outcome (rate ratio $=3.8 ; 95 \%$ $\mathrm{Cl}, 1.2-12.1)$. Patients with good and intermediate collaterals who did not achieve recanalization and patients with poor collaterals, even if they achieved recanalization, did not do well.

CONCLUSIONS: Patients with good or intermediate collaterals on CTA benefit from intra-arterial therapy, whereas patients with poor collaterals do not benefit from treatment.

ABBREVIATIONS: IAT = intra-arterial therapy; $r L M C=$ regional leptomeningeal collateral score; $S D=$ standard deviation

eptomeningeal collaterals are pre-existing anastomoses that connect a small number of distal-most arterioles within the crowns of the cerebral artery trees. ${ }^{1,2}$ During an acute stroke, ischemic brain depends on blood flow from these collaterals to survive until the occluded artery is opened. ${ }^{3-8}$ This collateral circulation is highly variable and potentially influences the rate at which an infarct grows. ${ }^{4,8-10}$ Collateral status at baseline is an independent determinant of clinical outcome among patients with acute ischemic stroke. , $5,7,8,11$ Nonetheless, "effect modification" by collat-

Received July 19, 2013; accepted after revision September 29.

From the Calgary Stroke Program, Departments of Clinical Neurosciences (V.N., M.A.A., S.M., E.Q., M.E., A.M.D., M.G., M.D.H., B.K.M.), Radiology (M.E., A.M.D., M.G., M.D.H., B.K.M.), and Community Health Sciences (M.D.H., B.K.M.), University of Calgary, Calgary, Alberta, Canada; Departments of Neurology (S.I.S.) and Radiology (H.W.C.), Brain Research Institute, Keimyung University, Daegu, South Korea; Department of Internal Medicine (M.A.A.), King Abdulaziz University, Jeddah, Saudi Arabia; and Hotchkiss Brain Institute (A.M.D., M.G., M.D.H., B.K.M.), University of Calgary, Calgary, Alberta, Canada.

Please address correspondence to Bijoy K. Menon, MD, 1079 A, 29th Street NW, Calgary, AB, Canada T3H4J2; e-mail: docbijoymenon@gmail.com

http://dx.doi.org/10.3174/ajnr.A3817 eral status measured noninvasively by use of CTA of the relationship between recanalization and clinical outcome has not been demonstrated before. This tool can be used to select patients for intra-arterial therapy (IAT) through demonstration of a differential clinical response to recanalization by collateral status.

In this study, we first demonstrate the concept of validity of collateral status measured by use of CTA among patients presenting with acute ischemic stroke by correlating it with infarct volume on baseline MR DWI and infarct growth over 24 hours. We then demonstrate "effect modification" by collateral status of the relationship between recanalization and clinical outcome in patients with acute ischemic stroke undergoing IAT, thus justifying the use of baseline collateral status on CTA as a patient selection tool for IAT.

\section{MATERIALS AND METHODS}

The Keimyung Stroke Registry is an ongoing, single-center, prospective cohort study of patients with acute ischemic stroke presenting to the Keimyung University, Dongsan Hospital in Daegu, South Korea. All patients undergo an NCCT of the head at admission followed by CTA of the head and neck. For the study (time period, May 2004 to 
July 2009), we included only patients presenting with acute ischemic stroke with M1 segment MCA with or without ICA occlusion on baseline CTA with witnessed stroke symptom onset who were treated with IAT and had a pretreatment brain MR imaging and 24-hour posttreatment MR imaging. During this time period, there were 286 patients with documented anterior circulation occlusions on baseline CTA. We excluded 202 patients (43 patients with a documented MCA occlusion beyond the M1 MCA segment, 28 patients with isolated ICA without M1 MCA occlusion, 7 patients with simultaneously detected occlusions in the posterior circulation or contralateral ICA territory, 71 patients with unknown stroke onset time, 16 patients with baseline MR imaging obtained after treatment, 29 patients with poor-quality MR imaging at baseline, and 8 patients without 24-hour MR imaging). Finally, 84 patients were included in these analyses. Stroke severity was assessed by use of the NIHSS at baseline, immediately after treatment, at discharge, and at 90 days. Interval times from stroke symptom onset to presentation in the emergency department, imaging, and endovascular procedures were collected. Functional status was assessed at baseline and 90 days by use of the mRS. We collected data on mRS by clinical review in a face-to-face interview at 3 months (in approximately $70 \%$ of patients) and by telephone interview at 3 months (in approximately $25 \%$ of patients). For $5 \%$ of patients, we obtained the 3-month mRS information at 5-9 months. The study was approved by the local institutional review board.

\section{Imaging Protocol}

Standard nonhelical NCCT was performed on a multisection scanner (Sensation 16; Siemens, Erlangen, Germany) by use of $120 \mathrm{kV}$, $170 \mathrm{mAs}$, with 5-mm section thickness. NCCT was followed by CTA with the use of a helical scan technique. Coverage was from arch to vertex, with continuous axial sections parallel to the orbitomeatal line with $0.6-1.25-\mathrm{mm}$ section thickness. Acquisitions were obtained after a single bolus intravenous contrast injection of $90-120 \mathrm{~mL}$ nonionic contrast media into an antecubital vein at $3-5 \mathrm{~mL} / \mathrm{s}$, auto-triggered by appearance of contrast in a region of interest manually placed in the ascending aorta. This was followed by a baseline MR imaging (3T Signa Excite; GE Healthcare, Milwaukee, Wisconsin) consisting of DWI, time-of-flight MRA, and gradient recalled-echo as a part of a stroke protocol. Follow-up MR imaging was performed within 24 hours by use of the same protocol.

\section{Intra-Arterial Therapy}

Intravenous tPA was given to all eligible patients within 3 hours of stroke onset as per accepted guidelines. MR imaging was primarily used to assess intracerebral hemorrhage on gradient recalledecho and recanalization status on MR TOF. Patients were taken to the angiography suite from the MR imaging suite for IAT. Patients with MCA occlusions with or without ICA occlusions who did not show recanalization on MRA or rapid clinical improvement after IV tPA were considered for IAT. IAT included local urokinase (10,000-20,000 IU/min; maximum permissible dose, 300,000 IU; Green Cross Pharm, Seoul, Republic of Korea), balloon angioplasty, or wire manipulation. Glycoprotein IIb/IIIa receptor antagonists were given in a small number of patients. For clots not manageable by the above methods, we used off-label stent placement (with the use of coronary stents). Recanalization was as- sessed by means of angiography in all cases. Successful recanalization was defined as TICI $2 \mathrm{~b} / 3$ flow on final angiogram. Penumbra Stroke System aspiration catheters (Penumbra, Alameda, California) and stent retriever devices (eg, Solitaire FR [Covidien, Irvine, California], Trevo [Stryker, Kalamazoo, Michigan]) were not available during this period.

\section{Imaging Analyses}

Baseline and follow-up images were analyzed at the imaging core lab of the Calgary Stroke Program. OsiriX version 3.5 (http:// www.osirix-viewer.com), an image-processing software designed for multiplanar reconstruction, was used to reconstruct $2 \mathrm{D}$ multiplanar reconstruction images of CTA in axial, coronal, and sagittal planes by use of 24-mm-thick slabs. Leptomeningeal collaterals were assessed on baseline CTA by consensus (B.K.M., S.I.S.) by use of the regional leptomeningeal score (rLMC), a previously published ordinal scoring system that is based on the Alberta Stroke Program Early CT Score template that has excellent interrater reliability ${ }^{3}$ (Fig 1). Infarct volumes were measured on baseline and 24-hour MR DWI by use of an in-house-validated software, Quantomo (Cybertrial, Calgary, Canada). Infarct growth over 24 hours was calculated by subtracting initial infarct volume from the 24-hour volume measurement. Quantomo has been previously validated and has good interrater and intrarater agreement. ${ }^{12}$ Readers were blinded to all clinical information and follow-up data at the time of scan reading.

\section{Statistical Analyses}

Continuous variables are summarized as means ( $\pm 1 \mathrm{SD})$ or medians (interquartile range or range) as appropriate. Collateral status by use of the rLMC score (0-20) was trichotomized into 3 groups: good (17-20), intermediate (11-16), and poor (0-10), according to previously published literature. ${ }^{3}$ Differences between these 3 groups were assessed by means of the Fisher exact test for proportions, 1-way ANOVA for parametric data, and a rank sum test for nonparametric data. We adjusted for multiple comparisons by use of the Bonferroni method. We tested for trend in outcome measure (infarct growth and mRS $0-2$ at 90 days) by collateral status in the recanalizers (TICI $2 \mathrm{~b}-3$ ) and in the non-recanalizers (TICI $0-1$ ) by use of the Cuzick test of trends. We then built a multivariable model by use of generalized linear modeling with a log link, with mRS $0-2$ versus $3-6$ at 90 days as the outcome and collateral status (good, intermediate, and poor), recanalization status (TICI $2 \mathrm{~b}-3$ versus $0-2 \mathrm{a}$ ), and age (by decile) as independent variables. Because there was no significant association of sex, baseline NIHSS, or onset to imaging time with $\mathrm{mRS}$ at 90 days, these variables were not included in the model. We specifically included a multiplicative interaction term between collateral status and recanalization status. All hypothesis tests were 2 -sided, with a value of $P<.05$ considered statistically significant. In multivariable analysis, interaction effects were considered significant at a value of $P<.10 .{ }^{13}$ Analyses were performed with the use of STATA/SE 12.1 software (StataCorp, College Station, Texas).

\section{RESULTS}

Among 84 patients, the mean age was $65.2 \pm 13.2$ years, median NIHSS score was 14 (interquartile range, 8.5), and median time 


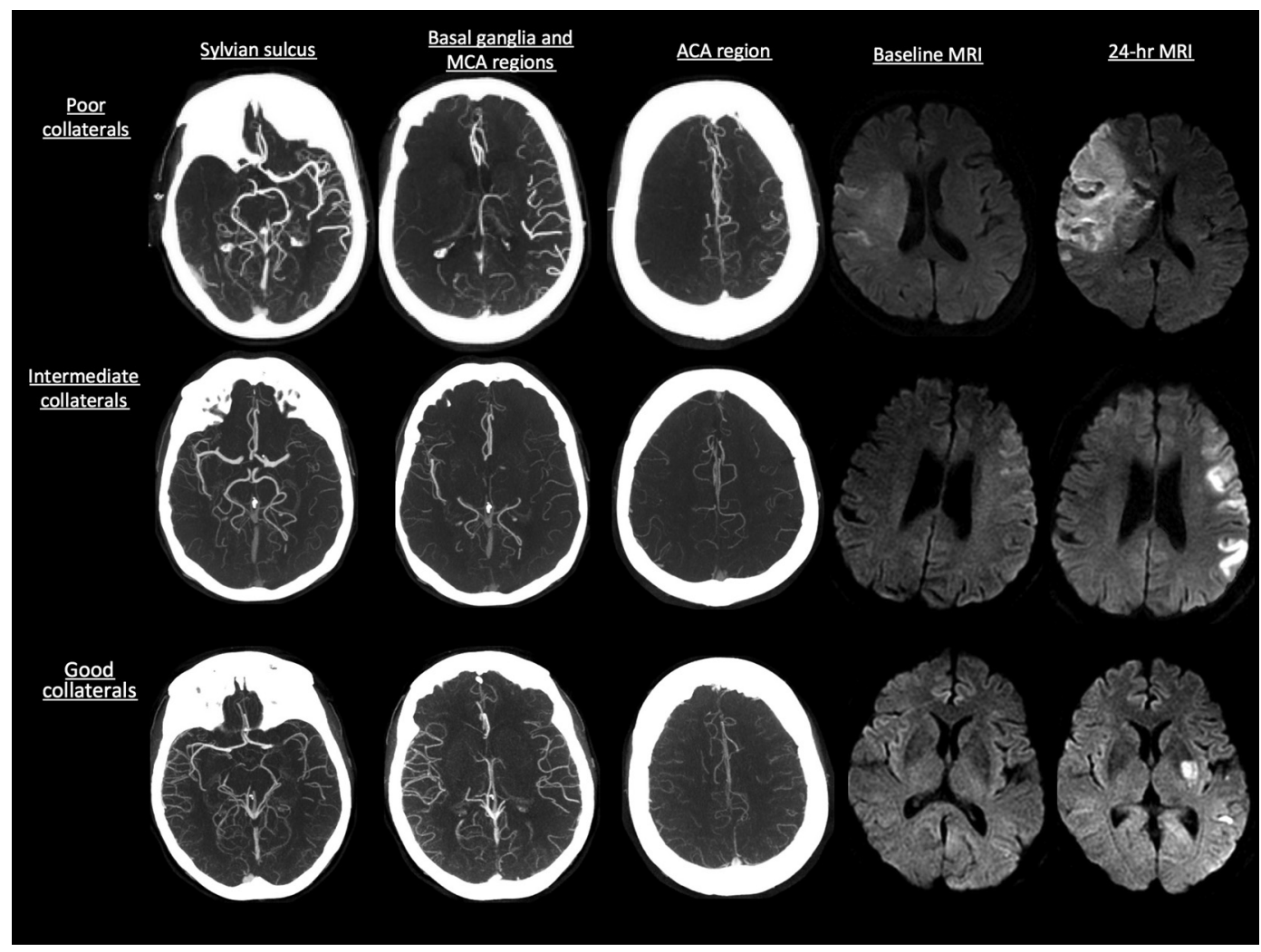

FIG 1. Collateral status on CTA as measured by the rLMC score with baseline and 24-hour infarct volume on MR DWI.

Table 1: Baseline characteristics of patients in study stratified by collateral status $(n=84)$

\begin{tabular}{|c|c|c|c|c|}
\hline & $\begin{array}{c}\text { Good Collaterals } \\
\text { (rLMC Score 17-20, } n=19 \text { ) }\end{array}$ & $\begin{array}{l}\text { Intermediate Collaterals } \\
\text { (rLMC Score } 11-16, n=34 \text { ) }\end{array}$ & $\begin{array}{c}\text { Poor Collaterals } \\
\text { (rLMC Score 0-10, } n=31 \text { ) }\end{array}$ & $P$ Value \\
\hline Age, $y$, median (range) & $63(26-78)$ & $66(40-85)$ & $70(45-89)$ & .15 \\
\hline Sex, male, \% & $52.6 \%$ & $58.8 \%$ & $45.2 \%$ & .55 \\
\hline Baseline NIHSS score, median (range) & $10(5-25)$ & $13(4-23)$ & $18(8-25)$ & $<.01$ \\
\hline Baseline ASPECTS, median (range) & $8(3-10)$ & $8(3-10)$ & $5(1-10)$ & $<.01$ \\
\hline Onset to baseline MRI time, min, median (range) & $166(68-724)$ & $176.5(55-493)$ & $148(36-334)$ & .06 \\
\hline Baseline infarct volume, $\mathrm{mL}( \pm 1 \mathrm{SD})$ & $12.6(13.2)$ & $37.1(32.1)$ & $110.1(75.1)$ & $<.01$ \\
\hline IV tPA (\%) & $52.6 \%$ & $55.9 \%$ & $48.4 \%$ & .72 \\
\hline Recanalization, \%, $\mathrm{TICl} 2 \mathrm{~b}-3$ & $31.6 \%$ & $50 \%$ & $29 \%$ & .2 \\
\hline Onset to recanalization, $\min ,{ }^{a} n=32$, median (range) & $400(295-766)$ & $360(160-620.5)$ & $290(157-323)$ & .01 \\
\hline Infarct growth, $\mathrm{mL}( \pm 1 \mathrm{SD})$ & $42.1(52.1)$ & $37.6(54.8)$ & $90.9(86.7)$ & $<.01$ \\
\hline
\end{tabular}

a In recanalizers alone $(n=32)$.

from stroke onset to initial MR imaging was 164 minutes. Successful recanalization (TICI $2 \mathrm{~b}-3$ ) was achieved in $38.1 \%$ of patients and good clinical outcome (mRS 0-2 at 90 days) in $35.8 \%$ of patients. Mean baseline infarct volume was $58.5 \mathrm{~mL}( \pm 1 \mathrm{SD}$, $64.4 \mathrm{~mL}$ ) and mean infarct growth over 24 hours was $58.3 \mathrm{~mL}( \pm 1$ $\mathrm{SD}, 72 \mathrm{~mL}$ ). There were 19 patients with good, 34 with intermediate, and 31 with poor collateral scores at baseline. Baseline characteristics stratified by good, intermediate, and poor collateral status are described in Table 1. Patients with good collateral status had lower baseline NIHSS score, higher baseline ASPECTS on NCCT, smaller baseline infarct volume on MR imaging, and lesser infarct growth over 24 hours (Table 1).

Infarct growth over 24 hours was significantly lower in patients with good collateral status at baseline who achieved recanalization $(7.0 \mathrm{~mL} ; \pm 1 \mathrm{SD}=11.7 \mathrm{~mL})$ when compared with those with intermediate collateral status who achieved recanalization $(26.6 \mathrm{~mL} ; \pm 1 \mathrm{SD}=43.4 \mathrm{~mL})$ and those with poor collaterals who recanalized $(67.7 \mathrm{~mL} ; \pm 1 \mathrm{SD}=75.1 \mathrm{~mL})(P=.05$; between group difference). There was no difference in infarct growth in the nonrecanalizers stratified by collateral status (good collaterals: 58.4 $\mathrm{mL}, \pm 1 \mathrm{SD}=58.6 \mathrm{~mL}$; intermediate collaterals: $48.6 \mathrm{~mL}, \pm 1$ $\mathrm{SD}=63.7 \mathrm{~mL}$; poor collaterals: $100.4 \mathrm{~mL}, \pm 1 \mathrm{SD}=91 \mathrm{~mL} ; P=$ .09 , between-group difference) (Fig 2). Similarly, good clinical outcome (mRS 0-2 at 90 days) was higher among patients with good collateral status who achieved recanalization (100\%) when compared with those with intermediate collateral status who recanalized $(58.8 \%)$ and those with poor collaterals who recanalized $(33.3 \%)(P=.04)$. There was no statistically significant difference in good clinical outcome in the non-recanalizers stratified by collateral status $(30.8 \%$ in those with good collaterals, $17.6 \%$ in those with intermediate collaterals, and $18.2 \%$ in those with poor collaterals; $P=.67)$. The Cuzick test of trend by collateral status was statistically significant for good clinical outcome ( $\mathrm{mRS} 0-2$ at 90 days, $P=.01$ ) in the recanalizers. There was no statistically significant trend by collateral status for good clinical outcome $(P=.08)$ in the non-recanalizers (Fig 3$)$. 


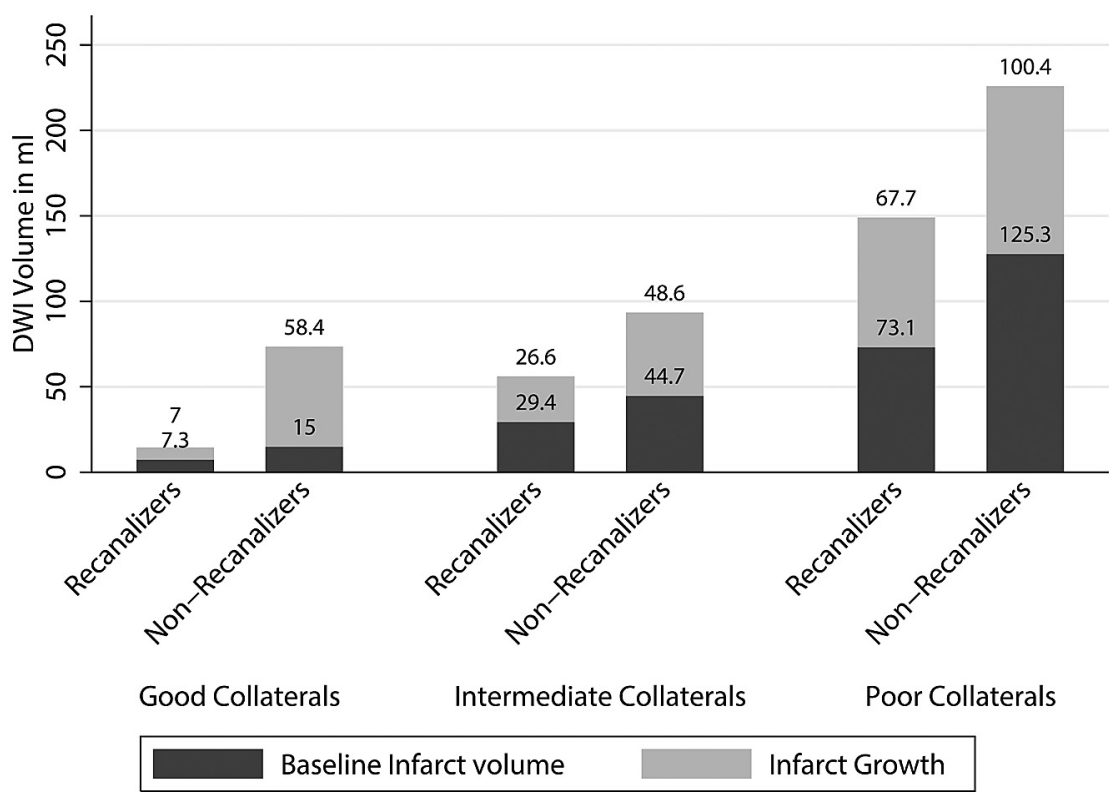

FIG 2. Mean baseline infarct volume and infarct growth over 24 hours stratified by baseline collateral status on CTA and final recanalization (TICI score $2 \mathrm{~b}-3$ versus $0-2 a$ ). time to screen, perform, and interpret. ${ }^{19}$ Many patients do not tolerate it as well as CT; image quality is affected by patient motion. MR imaging also has limited availability "after hours." ${ }^{20}$ Moreover, the recent MR Rescue Trial showed that the tool may not help in selecting patients for IAT. $^{21}$ NCCT ASPECTS has moderate interrater reliability, even among experts. ${ }^{22-25}$ Reliability is less in the early presenters (within 90 minutes from stroke symptom onset). ${ }^{26}$ In addition, ASPECTS interpretation is affected by patient motion and in the aged. ${ }^{27}$ CT perfusion needs algorithms for postprocessing images that are vendor-specific, not standardized, and therefore variable across centers. ${ }^{28-31}$ Trained personnel are sometimes needed to process these images. In addition, image quality is affected to some extent by patient motion. ${ }^{29}$ Additional radiation dose is also
In the multivariable model, the interaction between collateral status and recanalization was relevant $(P=.08)$. This final model also included age as a significant independent predictor of good clinical outcome. Given the presence of a statistically significant interaction in the model, we report age- and sexadjusted rate ratios for patients in 4 groups, namely, group 1 (poor collaterals who do not recanalize, $n=22$ ), group 2 (poor collaterals who recanalize, $n=9$ ), group 3 (intermediate or good collaterals who do not recanalize, $n=30$ ), and group 4 (intermediate or good collaterals who recanalize, $n=23$ ). $\mathrm{Pa}$ tients with intermediate and good collaterals were collapsed into 1 group for ease of reporting interaction effects. Only patients with intermediate or good collaterals who recanalize showed a statistically significant association with good clinical outcome (rate ratio $=3.8 ; 95 \% \mathrm{CI}, 1.2-12.1$ ). None of the other groups did as well clinically (Table 2). Fig 3 shows the unadjusted mRS scores at 90 days in these 4 groups stratified by recanalization status.

\section{DISCUSSION}

Our results show that collateral status measured at baseline by use of CTA among patients with acute ischemic stroke correlates with baseline infarct volume and with infarct growth over 24 hours. Furthermore, we demonstrated effect modification by collateral status of the relationship between recanalization and good clinical outcome. Patients with good and intermediate collaterals who achieve recanalization with IAT do well when compared with those who do not achieve recanalization. Patients with poor collaterals do not do well even if recanalization is achieved with IAT (Table 2).

Several imaging paradigms propose to "select" patients most suitable for IAT. ${ }^{14-18} \mathrm{MR}$ imaging-based diffusion/perfusion mismatch is one such paradigm. MR imaging, however, takes a concern. ${ }^{32}$ Collateral assessment on CTA has good interrater reliability. ${ }^{3,4,33}$ Good correlation with baseline infarct volume and infarct growth on MR DWI in our study demonstrates the tool's content validity. Furthermore, by demonstrating "effect modification" by collateral status among patients undergoing IAT, we demonstrate for the first time that this tool can be used to select patients for this therapy. Collateral assessment on CTA does not need any sophisticated algorithm or trained personnel for postprocessing images and is relatively resistant to patient motion-induced artifacts. It is available 24 hours a day in most centers. We therefore propose the use of this tool for patient selection within future endovascular trials.

Despite the variability in measuring collaterals at baseline in patients with acute ischemic stroke, our study further reinforces evidence from previous literature that patients with good collaterals at baseline have small baseline infarcts when compared with patients with intermediate and poor baseline collaterals. ${ }^{34,35} \mathrm{Re}$ canalization helps reduce further infarct growth, thus limiting size of final infarct. ${ }^{8}$ Our study also shows that the rate of infarct growth is quicker in patients with intermediate collaterals; these patients may only benefit if recanalization is achieved quickly. Patients with poor collaterals at baseline grow their infarcts the quickest; the likelihood that they will benefit from recanalization is the least. Nonetheless, the fact that some patients with poor collaterals in our study achieved good clinical outcome raises the possibility that routine CTA may have underestimated good and intermediate collaterals in some patients, misclassifying these collaterals as poor. Routine CTA is a single snapshot of contrastfilled blood vessels. Early timing of image acquisition with respect to that of bolus injection could potentially result in underestimation of true collateral status by use of this technique. ${ }^{3}$ The 4 patients who achieved good clinical outcome despite having poor collaterals at baseline and not achieving recanalization had a mean baseline infarct volume of $63.1 \mathrm{~mL}$ (range, 13.3-133.5 mL) 
Intermediate and Good

Collaterals

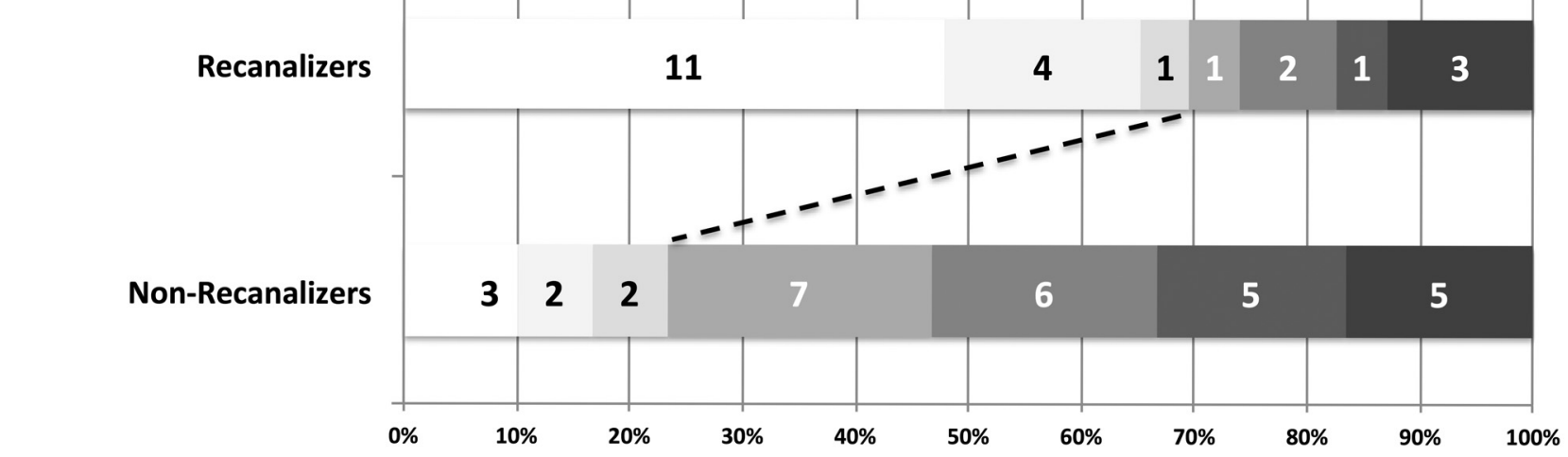

\section{Poor Collaterals}

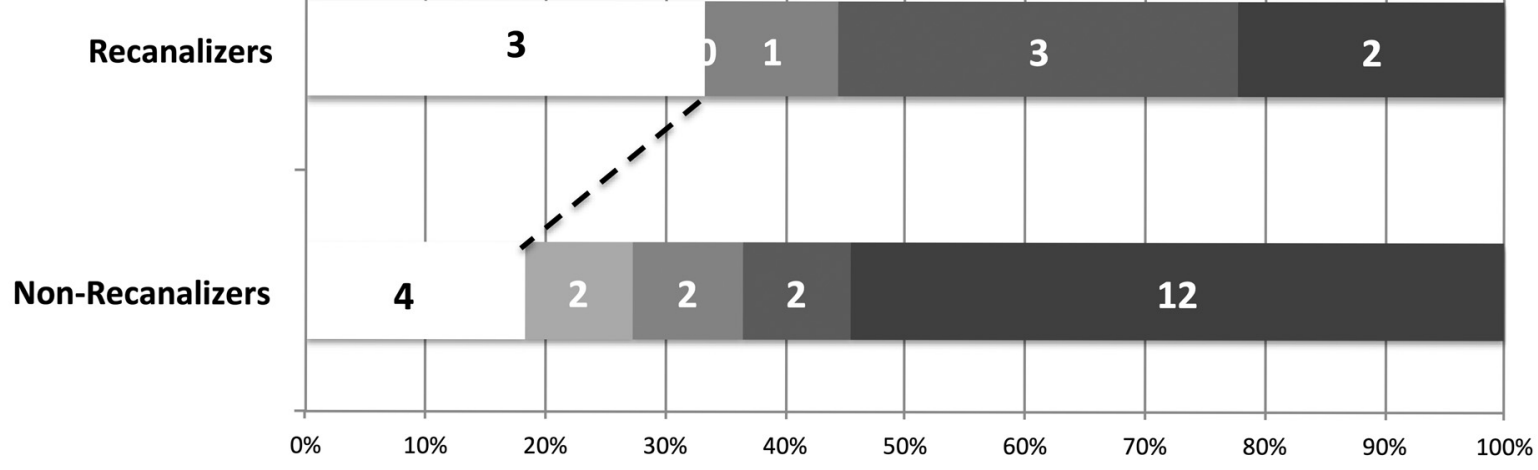

FIG 3. Ninety-day clinical outcome according to the mRS stratified by baseline collateral status on CTA and final recanalization (TICI score, $2 b-3)$.

Table 2: Generalized linear model with the use of log link showing rate ratios for good clinical outcome (mRS 0-2 at 90 days) by collateral status and recanalization status adjusted for age and sex

\begin{tabular}{|c|c|c|c|c|}
\hline Group & Rate Ratio & $95 \% \mathrm{Cl}$ & mRS 0-2 at 90 Days, $\%$ & $P$ Value \\
\hline Poor collaterals, non-recanalizers $(n=22)$ & 1 & - & $18.2 \%$ & - \\
\hline Poor collaterals, recanalizers $(n=9)$ & 2 & $0.5-8.3$ & $33.3 \%$ & .34 \\
\hline Intermediate and good collaterals, non-recanalizers $(n=30)$ & 1.6 & $0.5-5.5$ & $23.3 \%$ & .44 \\
\hline Intermediate and good collaterals, recanalizers $(n=23)$ & 3.8 & $1.2-12.1$ & $70 \%$ & .02 \\
\hline Age (for every 10 y below 80 ) & 1.2 & $1.1-1.4$ & - & .01 \\
\hline Sex, male & 0.6 & $0.4-1.1$ & & .12 \\
\hline
\end{tabular}

Note:-Reference group is patients with poor collaterals who do not recanalize.

compared with a mean baseline infarct volume of $110 \mathrm{~mL}$ for all patients with poor collaterals in the study, thus suggesting possible misclassification of good and intermediate collateral status as poor by use of routine CTA in at least those patients with small or intermediate baseline infarct volume. ${ }^{36}$ The rLMC score used in our study, being more liberal when defining poor collaterals in comparison with stricter definitions used in other studies, could also explain why some patients with poor collaterals did well in our study. ${ }^{34}$ Finally, variability in brain eloquence or possible misclassification of 90-day clinical outcome as the result of telephone follow-up in approximately $25 \%$ of patients could potentially explain why 2 patients with poor baseline collaterals and baseline infarct volumes $>80 \mathrm{~mL}$ in our study did well clinically. ${ }^{36}$ Both these patients had right hemisphere infarcts. Our study, however, is able to show with a degree of statistical certainty that the patients most likely to benefit from recanalization are those with good and intermediate baseline collaterals; patients with poor collaterals do not show a differential response to recanalization.

Reperfusion-related edema and injury has been postulated to be a cause for increased infarct growth among patients without mismatch who achieve reperfusion. ${ }^{18}$ In our study, patients with poor collateral status who did not recanalize had more infarct growth than did patients with poor collateral status who recanalized. Our results do not support the reperfusion injury hypothesis. On the contrary, we speculate that early recanalization, even among patients with poor collaterals, may reduce the risk of death. $^{37}$

Our study is not a randomized, controlled trial. Similar to previous studies supporting the use of a "mismatch"-based para- 
digm on perfusion imaging, our study provides evidence for the use of a "CTA collateral assessment-based paradigm" in selecting patients for IAT. ${ }^{18} \mathrm{We}$ did not have data on every procedural time metric; approximately 30\% of follow-up clinical data (mRS at 90 days) was ascertained by telephone. We were also underpowered to do a secondary analysis on the effect of time to recanalization on clinical outcomes stratified by collateral status. Our recanalization rates are modest and reflective of the time period when patients were recruited; nonetheless, our study is unique in that we were able to show a statistically significant interaction between collateral status and recanalization. With the advent of stent retrievers that achieve recanalization rates in excess of $80 \%$, future demonstration of such statistically significant "effect modification" may need very large cohorts. ${ }^{37}$ Our study is thus timely in being able to demonstrate the utility of this tool in patient selection for IAT.

\section{CONCLUSIONS}

The results from our study suggest a differential clinical and imaging response to recanalization by IAT in patients with good and intermediate collaterals. Patients with poor collaterals show no such differential response. Collateral assessment on CTA can potentially help aid patient selection for acute intra-arterial stroke treatment and for potential inclusion in endovascular stroke trials.

Disclosures: Emmad Qazi-UNRELATED: Employment: The Calgary Stroke Program, Comments: I work at the Calgary Stroke Program as a part-time employee. Mayank Goyal-UNRELATED: Consultancy: Covidien/ev3, Comments: For designing and conducting trial; Grants/Grants Pending: Covidien/ev3,* Comments: Funding for ESCAPE trial; Payment for Lectures (including service on speakers bureaus): Covidien/ev3, Comments: For speaking engagements related to acute stroke treatment. Michael Hill—UNRELATED: Board Membership: DSMB Aldagen trial; Grants/Grants Pending: Covidien, ${ }^{*}$ Comments: Grant for a clinical trial; Stock/Stock Options: Calgary Scientific Inc. (stock). Bijoy Menon—UNRELATED: Grants/Grants Pending: Heart and Stroke Foundation of Canada, ${ }^{*} \mathrm{CIHR}$. Andrew Demchuk (UNRELATED): Grant support and lecture fees: Covidien, ${ }^{*}$ Heart and Stroke Foundation of Canada, ${ }^{*}$ $\mathrm{ClHR}^{*}$. ('money paid to institution).

\section{REFERENCES}

1. Liebeskind DS. Collateral circulation. Stroke 2003;34:2279-84

2. Shuaib A, Butcher K, Mohammad AA, et al. Collateral blood vessels in acute ischaemic stroke: a potential therapeutic target. Lancet Neurol 2011;10:909-21

3. Menon BK, Smith EE, Modi J, et al. Regional leptomeningeal score on CT angiography predicts clinical and imaging outcomes in patients with acute anterior circulation occlusions. AJNR Am J Neuroradiol 2011;32:1640-45

4. Menon BK, O’Brien B, Bivard A, et al. Assessment of leptomeningeal collaterals using dynamic CT angiography in patients with acute ischemic stroke. J Cereb Blood Flow Metab 2013;33:365-71

5. Miteff F, Levi CR, Bateman GA, et al. The independent predictive utility of computed tomography angiographic collateral status in acute ischaemic stroke. Brain 2009;132:2231-38

6. Maas MB, Lev MH, Ay H, et al. Collateral vessels on CT angiography predict outcome in acute ischemic stroke. Stroke 2009;40:3001-05

7. Lima FO, Furie KL, Silva GS, et al. The pattern of leptomeningeal collaterals on CT angiography is a strong predictor of long-term functional outcome in stroke patients with large vessel intracranial occlusion. Stroke 2010;41:2316-22

8. Christoforidis GA, Mohammad Y, Kehagias D, et al. Angiographic assessment of pial collaterals as a prognostic indicator following intra-arterial thrombolysis for acute ischemic stroke. AJNR Am J Neuroradiol 2005;26:1789-97

9. Bang OY, Saver JL, Kim SJ, et al. Collateral flow predicts response to endovascular therapy for acute ischemic stroke. Stroke 2011; 42:693-99

10. Menon BK, Smith EE, Coutts SB, et al. Leptomeningeal collaterals are associated with modifiable metabolic risk factors. Ann Neurol 2013;74:241-48

11. Kim JJ, Fischbein NJ, Lu Y, et al. Regional angiographic grading system for collateral flow: correlation with cerebral infarction in patients with middle cerebral artery occlusion. Stroke 2004;35:1340-44

12. Steffenhagen N, Campos CR, Poppe AY, et al. Reliability of measuring lesion volumes in transient ischemic attack and minor stroke. Stroke 2010;41:814-16

13. The NINDS t-PA Stroke Study Group. Generalized efficacy of t-PA for acute stroke: subgroup analysis of the NINDS t-PA Stroke Trial. Stroke 1997;28:2119-25

14. Demchuk AM, Menon B, Goyal M. Imaging-based selection in acute ischemic stroke trials: a quest for imaging sweet spots. Ann N Y Acad Sci 2012;1268:63-71

15. Campbell BC, Christensen S, Levi CR, et al. Cerebral blood flow is the optimal CT perfusion parameter for assessing infarct core. Stroke 2011;42:3435-40

16. Ebinger M, Iwanaga T, Prosser JF, et al. Clinical-diffusion mismatch and benefit from thrombolysis 3 to 6 hours after acute stroke. Stroke 2009;40:2572-74

17. Kakuda W, Lansberg MG, Thijs VN, et al. Optimal definition for PWI/DWI mismatch in acute ischemic stroke patients. J Cereb Blood Flow Metab 2008;28:887-91

18. Lansberg MG, Straka M, Kemp S, et al. MRI profile and response to endovascular reperfusion after stroke (DEFUSE 2): a prospective cohort study. Lancet Neurol 2012;11:860-67

19. Sheth KN, Terry JB, Nogueira RG, et al. Advanced modality imaging evaluation in acute ischemic stroke may lead to delayed endovascular reperfusion therapy without improvement in clinical outcomes. J Neurointervent Surg 2013;5 suppl 1:i62-65

20. Hand PJ, Wardlaw JM, Rowat AM, et al. Magnetic resonance brain imaging in patients with acute stroke: feasibility and patient related difficulties. J Neurol Neurosurg Psychiatry 2005;76:1525-27

21. Kidwell CS, Jahan R, Gornbein J, et al. A trial of imaging selection and endovascular treatment for ischemic stroke. $N$ Engl J Med 2013;368:914-23

22. Gupta AC, Schaefer PW, Chaudhry ZA, et al. Interobserver reliability of baseline noncontrast CT Alberta Stroke Program Early CT Score for intra-arterial stroke treatment selection. AJNR Am J Neuroradiol 2012;33:1046-49

23. Wardlaw JM, Mielke O. Early signs of brain infarction at CT: observer reliability and outcome after thrombolytic treatmentsystematic review. Radiology 2005;235:444-53

24. Patel SC, Levine SR, Tilley BC, et al. Lack of clinical significance of early ischemic changes on computed tomography in acute stroke. JAMA 2001;286:2830-38

25. Coutts SB, Hill MD, Demchuk AM, et al. ASPECTS reading requires training and experience. Stroke 2003;34:e179

26. Bal S, Bhatia R, Menon BK, et al. Time dependence of reliability of noncontrast computed tomography in comparison to computed tomography angiography source image in acute ischemic stroke. Int J Stroke 2012 Sep 13. [Epub ahead of print]

27. Barber PA, Demchuk AM, Zhang J, et al. Validity and reliability of a quantitative computed tomography score in predicting outcome of hyperacute stroke before thrombolytic therapy: ASPECTS Study Group: Alberta Stroke Programme Early CT Score. Lancet 2000;355:1670-74

28. Kudo K, Sasaki M, Yamada K, et al. Differences in CT perfusion maps generated by different commercial software: quantitative analysis by using identical source data of acute stroke patients. $R a$ diology 2010;254:200-09

29. Goyal M, Menon BK, Derdeyn CP. Perfusion imaging in acute ischemic stroke: let us improve the science before changing clinical practice. Radiology 2013;266:16-21

30. Zussman BM, Boghosian G, Gorniak RJ, et al. The relative effect of 
vendor variability in CT perfusion results: a method comparison study. AJR Am J Roentgenol 2011;197:468-73

31. Bivard A, Levi C, Spratt N, et al. Perfusion CT in acute stroke: a comprehensive analysis of infarct and penumbra. Radiology 2012;267:543-50

32. Mnyusiwalla A, Aviv RI, Symons SP. Radiation dose from multidetector row CT imaging for acute stroke. Neuroradiology 2009;51:635-40

33. McVerry F, Liebeskind DS, Muir KW. Systematic review of methods for assessing leptomeningeal collateral flow. AJNR Am J Neuroradiol 2012;33:576-82

34. Souza LC, Yoo AJ, Chaudhry ZA, et al. Malignant CTA collateral profile is highly specific for large admission DWI infarct core and poor outcome in acute stroke. AJNR Am J Neuroradiol 2012;33:1331-36
35. Tan IY, Demchuk AM, Hopyan J, et al. CT angiography clot burden score and collateral score: correlation with clinical and radiologic outcomes in acute middle cerebral artery infarct. AJNR Am J Neuroradiol 2009;30:525-31

36. Yoo AJ, Verduzco LA, Schaefer PW, et al. MRI-based selection for intra-arterial stroke therapy: value of pretreatment diffusionweighted imaging lesion volume in selecting patients with acute stroke who will benefit from early recanalization. Stroke 2009;40:2046-54

37. Saver JL, Jahan R, Levy EI, et al. Solitaire flow restoration device versus the Merci retriever in patients with acute ischaemic stroke (SWIFT): a randomised, parallel-group, non-inferiority trial. Lancet 2012;380:1241-49 